\title{
Photonic Generation and Distribution of Coherent Multiband THz Wireless Signals
}

\author{
Martyn Fice, Haymen Shams, Zhen Yang, Luis Gonzalez-Guerrero, \\ Michele Natrella, Cyril Renaud, and Alwyn Seeds \\ University College London, Department of Electronic and Electrical Engineering, Torrington Place, London, U.K. \\ m.fice@ucl.ac.uk
}

\begin{abstract}
We discuss photonic generation of high-speed THz wireless signals, with particular reference to how multiband signals could be distributed over fibre networks from a central baseband unit equipped with a pool of optoelectronic components, allowing the remote antenna unit to be very simple, while delivering flexibility in terms of data rate and $\mathrm{THz}$ carrier frequency. The proposed scheme is demonstrated experimentally by generating a 5-channel multiband signal with aggregate data rate of $100 \mathrm{~Gb} / \mathrm{s}$ and investigating the performance of each $20 \mathrm{~Gb} / \mathrm{s}$ sub-band after transmission over a wireless link in the $220-280 \mathrm{GHz}$ band.
\end{abstract}

Index Terms-THz communications, photonic heterodyning.

\section{INTRODUCTION}

The demand for wireless data transmission at ever higher rates, for applications such as video streaming, is driving investigation of wireless transmission at frequencies in the millimetre-wave and higher bands [1]. Spectrum is available at low $\mathrm{THz}$ frequencies $(200 \mathrm{GHz}-500 \mathrm{GHz})$ with potential bandwidths of tens of gigahertz, limited by the location of water absorption peaks at longer transmission distances (100 $\mathrm{m}$ upwards) and by practical considerations such as the frequency range of standard waveguides or regulatory requirements. Fundamental to operating at these frequencies is the extremely high free-space path loss (more than $100 \mathrm{~dB}$ for link lengths greater than $10 \mathrm{~m}$ ). When the low power of compact sources for these wavebands and the large noise bandwidth associated with high-baud-rate transmission are taken into consideration, highly directional antennas are essential to achieve an adequate signal-to-noise ratio at the receiver. Even so, most demonstrations of wireless data transmission at these low $\mathrm{THz}$ frequencies have been limited to short distances, a few centimetres to a few tens of metres.

To illustrate the challenges, Table 1 presents a power budget calculation for a wireless link at $300 \mathrm{GHz}$. A transmitter output power of $10 \mathrm{~mW}$ is assumed, based on the maximum reported output power of state-of-the-art InP highelectron-mobility transistor monolithic millimeter-wave integrated circuit (MMIC) electronic amplifiers at around this frequency [2]. Antenna gain for both transmitter and receiver is taken to be $25 \mathrm{dBi}$, typical of compact horn antennas, while the loss for downconversion to the IF is assumed to be $10 \mathrm{~dB}$, similar to the performance of commercially available sub-harmonic mixers based on Schottky diodes. This simplified calculation shows that there is around $5 \mathrm{~dB}$ margin for transmission of 10 Gbaud quadrature phase shift keying (QPSK) over a link length of $10 \mathrm{~m}$ with a bit error ratio (BER) of $10^{-3}$, thus allowing for some implementation penalty. Such a wireless system might be compatible with high data rate transmission to nomadic devices within an office, for example. Using more highly directional antennas (e.g. Cassegrain) with gain of $50 \mathrm{dBi}$ at both transmitter and receiver, a similar calculation shows that $100 \mathrm{~Gb} / \mathrm{s}$ QPSK could be transmitted with similar performance over more than $1 \mathrm{~km}$, which might be suitable for mobile front- or back-haul applications.

TABLE I. EXAMPLE THZ WIRELESS LiNK POWER BUDGET

\begin{tabular}{|l|c|c|l|}
\hline Parameter & Value & Unit & \multicolumn{1}{c|}{ Assumptions } \\
\hline Source power & 10 & $\mathrm{dBm}$ & \\
\hline Tx antenna gain & 25 & $\mathrm{dBi}$ & \\
\hline Transmission loss & 102 & $\mathrm{~dB}$ & $\begin{array}{l}\text { At } 300 \mathrm{GHz} \text {; link length }= \\
10 \mathrm{~m} \text {; absorption negligible }\end{array}$ \\
\hline Rx antenna gain & 25 & $\mathrm{dBi}$ & \\
\hline Received power & $\mathbf{- 4 2}$ & $\mathbf{d B m}$ & \\
\hline IF power & $\mathbf{- 5 2}$ & $\mathrm{dBm}$ & $10 \mathrm{~dB}$ down-conversion loss \\
\hline $\begin{array}{l}\text { IF input equivalent } \\
\text { noise }\end{array}$ & -167 & $\begin{array}{c}\mathrm{dBm} \\
/ \mathrm{Hz}\end{array}$ & $\mathrm{NF}=7 \mathrm{~dB}$ \\
\hline $\mathbf{E}_{\mathbf{b}} / \mathbf{N}_{\mathbf{0}}$ & $\mathbf{1 2}$ & $\mathbf{d B}$ & Data rate $=20 \mathrm{~Gb} / \mathrm{s}$ \\
\hline System margin & $\sim 5$ & $\mathrm{~dB}$ & $\begin{array}{l}\text { c.f. } \mathrm{BPSK} \text { or } \mathrm{QPSK} \text { at } \\
\text { BER }=10^{-3}\left(\mathrm{E}_{\mathrm{b}} / \mathrm{N}_{0}=6.8 \mathrm{~dB}\right)\end{array}$ \\
\hline
\end{tabular}

\section{PhOtONiC Generation OF THz SignAls}

Most experimental demonstrations of high-speed THzwireless data transmission have employed photonic generation [3 - 7], making use of optoelectronic components and sub-systems developed for optical fibre communications systems. High symbol rate $(10-30$ Gbaud $)$ signals can be modulated onto optical carriers in various formats, including on-off keying (OOK), QPSK and 16-QAM (quadrature amplitude modulation), and then downconverted to the $\mathrm{THz}$ band by heterodyning the modulated optical signal with a continuous wave laser (optical local oscillator, LO) on a high-speed photodiode (PD), which acts as a mixer. The critical component is the PD, which needs to respond at the $\mathrm{THz}$ frequency of interest. High bandwidth PDs, such as the uni-travelling carrier (UTC) PD, are required, but even so, at frequencies above $200 \mathrm{GHz}$ these are almost always operating beyond their $-3-\mathrm{dB}$ bandwidth. 
Due to the reduced PD response at high frequencies and the need to operate at relatively low photocurrents to avoid damage, the power generated photonically at $\mathrm{THz}$ frequencies is quite low. The maximum generated to date at $300 \mathrm{GHz}$ is $1 \mathrm{~mW}$, obtained by combining the output of two monolithically integrated UTC-PDs [8]. It is likely that electrical amplification of the PD output will be required to achieve significantly more transmitter power, although, as noted earlier, the output power of state-of-the-art amplifiers in the $300 \mathrm{GHz}$ band is itself currently $10 \mathrm{~mW}$ or less [2].

Photonic $\mathrm{THz}$ generation has been used to demonstrate transmission of a total of $100 \mathrm{~Gb} / \mathrm{s}$ in three sub-channels at a carrier frequency of $237.5 \mathrm{GHz}$, over a distance of $20 \mathrm{~m}$ [3]. A MMIC was used at the receiver to recover in-phase and quadrature signals, which were then digitised and processed offline to recover the data and to estimate the BER. An alternative receiver architecture is to use a sub-harmonic mixer (SHM), usually based on a high-speed Schottky barrier diode, to downconvert to an intermediate frequency (IF), which is then digitised and processed offline to recover the baseband data. This approach has been used to demonstrate $20 \mathrm{Gbaud}$ QPSK $(40 \mathrm{~Gb} / \mathrm{s})$ at $325 \mathrm{GHz}$ over $15 \mathrm{~m}$ [4], and $46 \mathrm{~Gb} / \mathrm{s}$ at $400 \mathrm{GHz}$ over $2 \mathrm{~m}$ [5]. Employing direct detection using an SHM, real-time, error-free transmission of $48 \mathrm{~Gb} / \mathrm{s}$ data has been achieved at $300 \mathrm{GHz}$, using polarisation multiplexing and OOK [6].

The link budget calculations described in the Introduction show that, unless extremely highly directional antennas are used, $\mathrm{THz}$ wireless transmission will be possible (at least in the foreseeable future) over only short distances, a few tens of metres at most. This implies that a large number of small cells would be needed to build a $\mathrm{THz}$ wireless mobile communications network, even in a relatively small area, such as a sports stadium. Alternatively, $\mathrm{THz}$ wireless might be used within a room, to deliver ultra-high-speed broadband access. Distribution of the high-speed data to the radio access point in each cell or room is likely to be done on optical fibre, and hence photonic generation of the THz-wireless signal directly from the optical signal is very attractive, because of the greatly simplified equipment required at the remote antenna unit (RAU), as illustrated in Fig. 1.

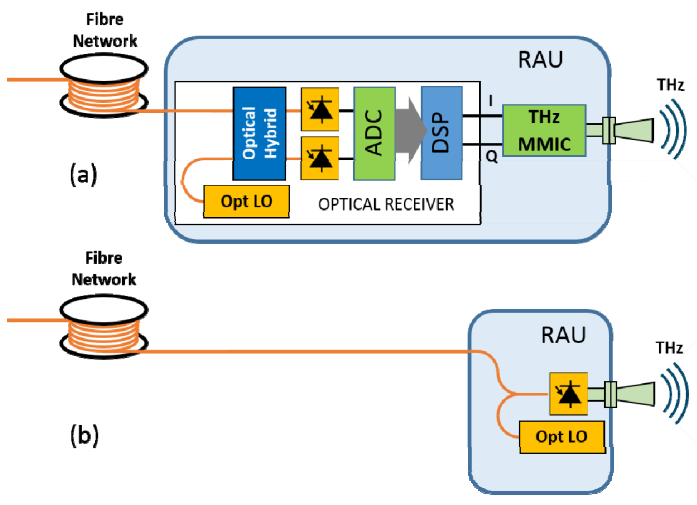

Fig. 1. Conversion of high-speed optical signals to $\mathrm{THz}$ signals using (a) optoelectronic detection followed by a THz MMIC upconverter, and (b) photonic heterodyning.

\section{MULTIBAND GENERATION AND DistRIBUTION}

The spectral windows being considered for $\mathrm{THz}$ wireless transmission are very wide, for instance IEEE 802.15 TG3d is considering the band from 252 to $325 \mathrm{GHz}$ (i.e. $73 \mathrm{GHz}$ bandwidth) [9]. Even assuming double sideband modulation, a single-carrier signal requiring the whole of this bandwidth is beyond the capabilities of photonic generation using the optoelectronic modulators typically employed in commercial optical communications systems. Furthermore, although a maximum data rate of $100 \mathrm{~Gb} / \mathrm{s}$ is being discussed [9], it is unlikely that all of the available bandwidth would be dedicated to one user to deliver this data rate. It seems more realistic to assume that the spectrum will be divided into subbands to deliver lower rate data (e.g. 10 or $20 \mathrm{~Gb} / \mathrm{s}$ ) to more than one user. Here, we propose a scheme for photonically generating and distributing multiband signals which offers great flexibility in terms of data rate and $\mathrm{THz}$ carrier frequency, while maintaining a simple RAU architecture. Our proposal builds on concepts being considered for variable bandwidth transceivers for software defined optical networks [10], and shares elements of optical fronthaul architectures.

The basic scheme is illustrated in Fig. 2. The baseband unit (BBU) includes a number of lasers at different wavelengths which may be reconfigurably connected to a bank of optical modulators through an optical space switch. The modulators may have different maximum baud rate capabilities, being selected to match the required baud rate for each sub-band. The outputs of several of the modulators are combined with one or more $\mathrm{CW}$ optical LO lasers (shown as tuneable lasers in Fig. 2) in an optical space / wavelength switch to form a multiband multiplex (as illustrated in the inset of Fig. 2) which is transmitted to a RAU over optical fibre. A passive optical network (PON) could be used to distribute the multiband signals for several RAUs situated close to each other. At the RAU, optical filtering is used to select the sub-band(s) and optical LO required for each antenna at the RAU, and converted to $\mathrm{THz}$ signals by heterodyning on a PD. This allows signals to be transmitted on the same or different frequencies from different antennas covering different sectors, or to support MIMO transmission.

The scheme may be extended by replacing the banks of carrier and LO lasers with an optical frequency comb generator (OFCG). This improves the frequency accuracy of the generated $\mathrm{THz}$ signals, since the carrier frequency is an integer multiple of the comb line spacing, which is determined by the frequency of the RF oscillator driving the OFCG, rather than being determined by the frequency difference between free-running lasers. In addition, the phase noise of the generated $\mathrm{THz}$ carrier is greatly reduced, since the optical comb lines are phase correlated. Although simulations show that digital signal processing (DSP) at the receiver using phase-estimation techniques based on the Viterbi-Viterbi algorithm can be used to track and correct for phase noise in the case of free-running lasers, giving demodulation with low penalty (Fig. 3), the coherent 
approach based on an OFCG could simplify the DSP, which could be important for low-power real-time systems, as well as ensuring that the carrier frequency is accurately defined.

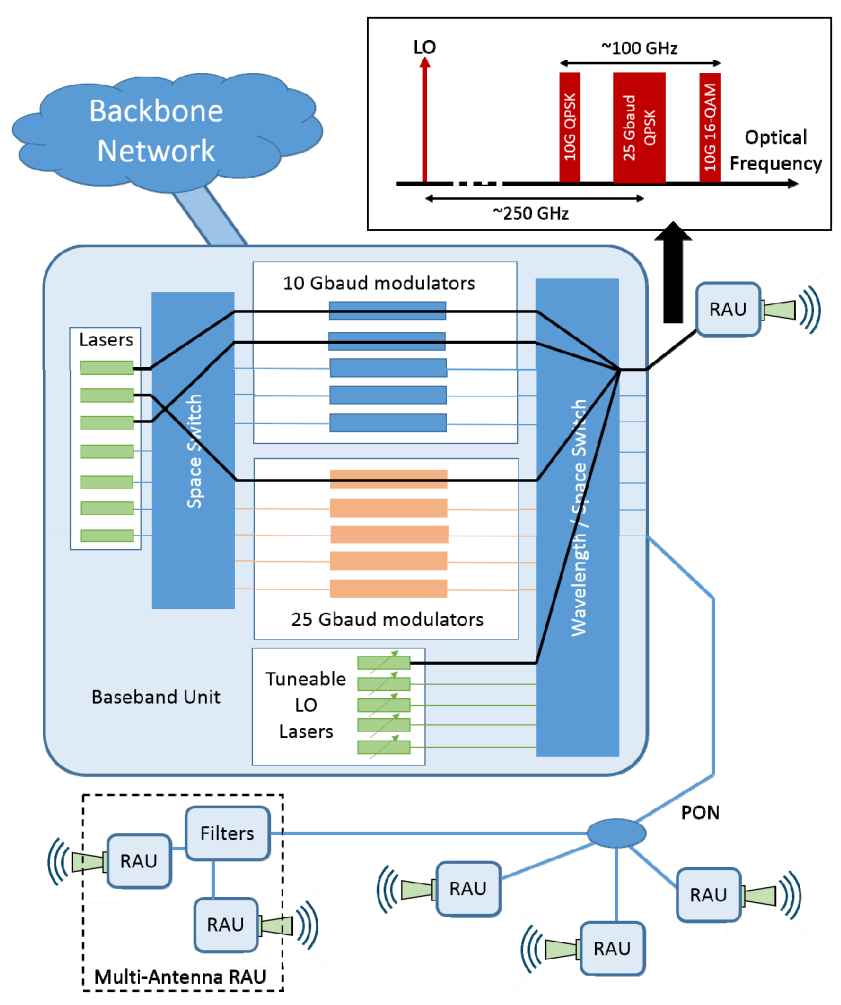

Fig. 2. Schematic of the proposed multiband photonic $\mathrm{THz}$ generation scheme. The inset shows the optical spectrum of one of the optical multiband signals that could be generated for distribution to a remote antenna unit (RAU) for conversion to a $\mathrm{THz}$ wireless signal.

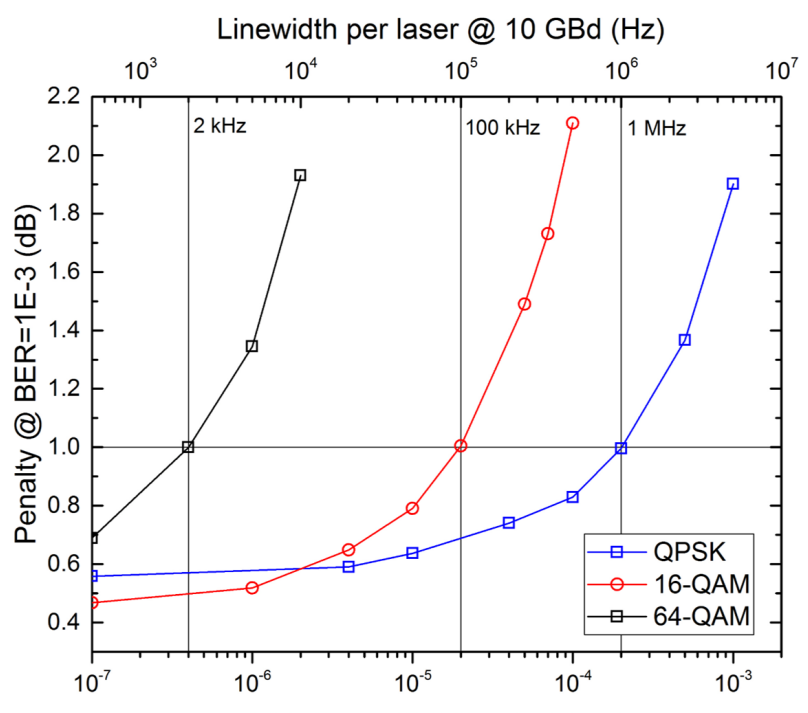

$(\mathrm{THz} \text { linewidth })^{*}($ Symbol time $)$

Fig. 3. Penalty as a function of normalised $\mathrm{THz}$ linewidth for different modulation formats, obtained by simulation, using an M-th power block algorithm for phase estimation at the receiver.
A drawback of transmitting the LO (or several LOs) from the BBU is that it is wasteful of optical spectrum, with only about half of the optical spectrum being available to transmit data from the BBU to RAUs in the case of $100 \mathrm{GHz}$ multibands and $250 \mathrm{GHz}$ carrier frequency, even if multiplexes for different RAUs are interleaved to improve spectral efficiency. Despite this, up to 20 RAUs, each fed with around $100 \mathrm{~Gb} / \mathrm{s}$ aggregate data, could be served using a single PON operating in the optical C-band (1530 $1565 \mathrm{~nm})$.

\section{EXPERIMENTAL DEMONSTRATION}

We have carried out an experimental demonstration to explore some of the key aspects of the multiband distribution scheme, using the arrangement shown schematically in Fig. 4. In this case a free-running LO laser was placed at the RAU, to allow a scheme for using the LO laser to transmit an upstream signal to the central office to be demonstrated as well [11]. However, we have previously demonstrated photonic $\mathrm{THz}$ generation using a line from a comb source, transmitted with the multiband signal, as the LO [7], and the phase coherence of the $\mathrm{LO}$ and signal carriers has also been investigated [12].

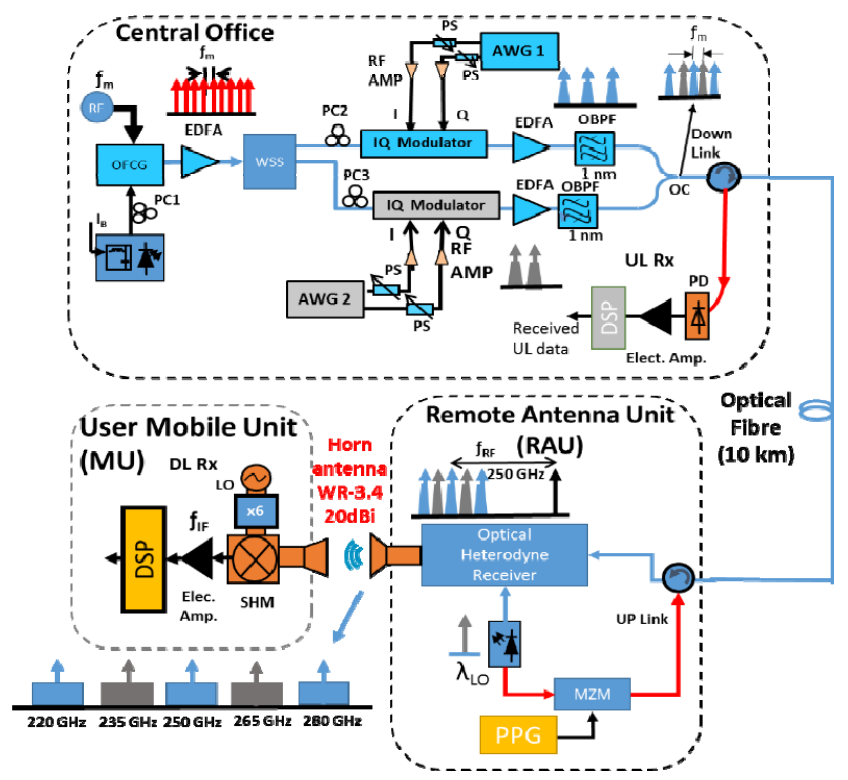

Fig. 4. Arrangement for the experimental demonstration of multiband photonic THz generation.

A narrow-linewidth $(10 \mathrm{kHz})$ laser with wavelength of $1550 \mathrm{~nm}$ was used as the seed for the OFCG, which is based on a dual-drive Mach-Zehnder modulator driven at $15 \mathrm{GHz}$, which sets the comb line spacing. Five lines from the comb were selected and divided into odd and even groups using a programmable optical filter and the groups separately modulated using two IQ optical modulators driven by arbitrary waveform generators (AWGs). Two pseudorandom bit sequences were used to drive each modulator to give 10 Gbaud QPSK modulation (20 Gb/s per sub-band), 
using root raised cosine (RRC) filters with a roll-off factor of 0.01 to limit the sub-bands to the Nyquist bandwidth $(10 \mathrm{GHz})$. The modulated optical signals were combined and transmitted over $10 \mathrm{~km}$ of standard single-mode optical fibre to the RAU.

At the RAU, the optical multiband signal was combined with a tuneable laser (optical LO) with a linewidth of $100 \mathrm{kHz}$, then amplified and optically filtered to select one of the data sub-bands and the optical LO. A THz signal was generated by heterodyning the filtered signal on a UTC PD and radiated from a $20 \mathrm{dBi}$ WR-3.4 horn antenna. Depending on the sub-band selected, the signal was centred on a frequency of $220,235,250,265$, or $280 \mathrm{GHz}$. The $\mathrm{THz}$ signal was received by another $20 \mathrm{dBi}$ WR-3.4 horn antenna and downconverted to an intermediate frequency (IF) of $12 \mathrm{GHz}$ by using a sub-harmonic mixer (SHM) operated with an electrical LO. The IF signal was amplified and digitised using a real-time scope with sampling rate $80 \mathrm{GSample} / \mathrm{s}$ and analogue bandwidth of $36 \mathrm{GHz}$.

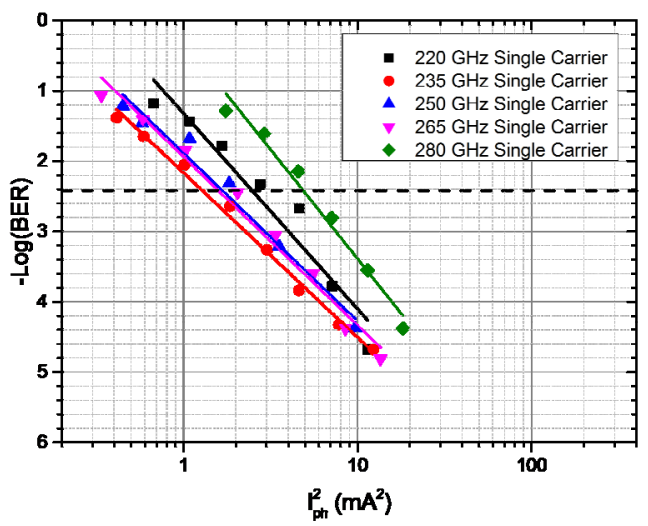

(a)

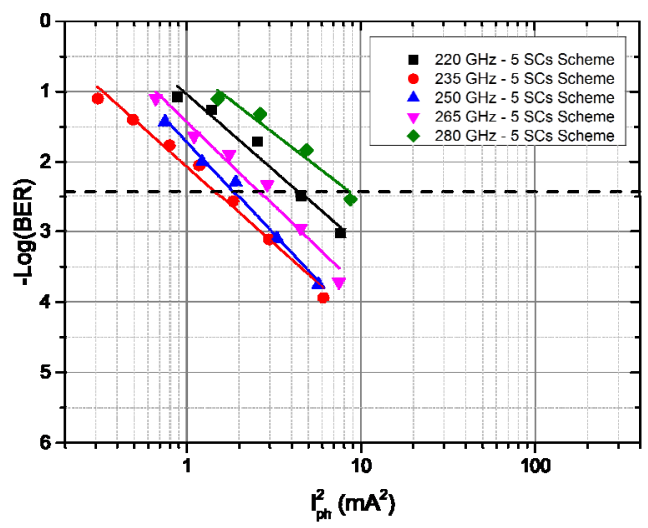

(b)

Fig. 5. Measured BER as a function of the photocurrent squared (proportional to $\mathrm{THz}$ power) for each sub-band after wireless transmission. In (a) the sub-bands are distributed as a single channel, and in (b) as part of a multiband signal.

The signal was recovered by offline DSP, starting by digital downconversion to baseband using the nominal value of the IF, followed by RRC filtering with 0.01 roll-off factor (to match the transmitter filter). Subsequent DSP steps included channel equalisation, carrier phase estimation, and BER calculation, as described in [13].

The system performance was first determined for each of the sub-bands transmitted over the fibre independently, and then with the multiband transmitted. The results are shown in Fig. 5. The BER is plotted against the square of the photocurrent, which is expected to be proportional to the received $\mathrm{THz}$ power for a fixed wireless link length. The single carrier results show some variation with frequency, with the $220 \mathrm{GHz}$ and $280 \mathrm{GHz}$ sub-bands showing penalties of a few $\mathrm{dB}$ compared to the other channels. This is due to the $220 \mathrm{GHz}$ channel being close to the lower operating frequency range of the WR-3.4 waveguide, and the UTC frequency response roll-off at $280 \mathrm{GHz}$. The results for the multiband show a similar trend, but with increased penalty compared to the $235 \mathrm{GHz}$ sub-band, due to the effect of optical filtering at the RAU.

In all cases, the minimum BER measured is well below $10^{-3}$, so could be corrected by forward error correction techniques. These measurements were made over a very short link $(\sim 2 \mathrm{~cm})$, but by using polymethylpentene lenses at both transmitter and receiver to collimate the $\mathrm{THz}$ emission, the transmission distance was increased to $70 \mathrm{~cm}$ (limited by the experimental arrangement), with a BER of approximately $2 \times 10^{-5}[11]$.

\section{CONCLUSION}

We have proposed a scheme for distributing multiband optical signals to remote antenna units (RAUs) where the optical signals are converted into $\mathrm{THz}$ wireless signals by photonic heterodyning on a high-speed photodiode. All the high-speed optoelectronic components for down-stream transmission are sited in a baseband unit remote from the RAUs, allowing resources to be reconfigured according to changing requirements, while keeping the RAUs simple. The scheme gives considerable flexibility in terms of the signal modulation format and baud rate, the number of subchannels in the multiband, and the $\mathrm{THz}$ carrier frequency. Key aspects of the proposed multiband photonic $\mathrm{THz}$ generation scheme have been demonstrated by generating a multiband with an aggregate data rate of $100 \mathrm{~Gb} / \mathrm{s}$ in five sub-bands. The performance of sub-band transmission in the $220-280 \mathrm{GHz}$ frequency band has been measured for directional links with lengths up to $70 \mathrm{~cm}$.

\section{ACKNOWLEDGMENT}

This work was supported in part by the UK Engineering and Physical Sciences Research Council under the Coherent TeraHertz Systems Programme Grant (EP/J017671/1), and by the European Commission through the European project iPHOS (grant agreement no: 257539). 


\section{REFERENCES}

[1] T. Nagatsuma, G. Ducournau, and C. C. Renaud, "Advances in terahertz communications accelerated by photonics," Nat. Photonics, vol. 10, no. 6, pp. 371-379, 2016.

[2] V. Radisic, W. R. Deal, K. M. K. H. Leong, X. B. Mei, W. Yoshida, Po-Hsin Liu, J. Uyeda, A. Fung, L. Samoska, T. Gaier, and R. Lai, "A $10-\mathrm{mW}$ submillimeter-wave solid-state power-amplifier module," IEEE Trans. Microw. Theory Tech., vol. 58, no. 7, pp. 1903-1909, Jul. 2010.

[3] S. Koenig, D. Lopez-Diaz, J. Antes, F. Boes, R. Henneberger, A. Leuther, A. Tessmann, R. Schmogrow, D. Hillerkuss, R. Palmer, T. Zwick, C. Koos, W. Freude, O. Ambacher, J. Leuthold, and I. Kallfass, "Wireless sub-THz communication system with high data rate," Nat. Photonics, vol. 7, no. 12, pp. 977-981, 2013.

[4] A. Kanno, T. Kuri, I. Morohashi, I. Hosako, T. Kawanishi, Y. Yoshida, K. Kitayama, "20-Gbaud QPSK coherent radio transmission at $325 \mathrm{GHz}$ with high-gain antennas," Conf. on Infrared, Millimeter, and Terahertz waves (IRMMW-THz), 2014.

[5] G. Ducournau, P. Szriftgiser, A. Beck, D. Bacquet, F. Pavanello, E. Peytavit, M. Zaknoune, T. Akalin, and J. Lampin, "Ultrawide bandwidth single channel $0.4 \mathrm{THz}$ wireless link combining broadband quasi-optic photomixer and coherent detection," IEEE Trans. Terahertz Sci. Technol., vol. 4, no. 3, pp. 328-337, 2014.

[6] T. Nagatsuma, S. Horiguchi, Y. Minamikata, Y. Yoshimizu, S. Hisatake, S. Kuwano, N. Yoshimoto, J. Terada, and H. Takahashi, "Terahertz wireless communications based on photonics technologies," Optics Express, vol. 21, no. 20 , pp. 23736-23747, 2013.

[7] H. Shams, T. Shao, M. J. Fice, P. M. Anandarajah, C. C. Renaud, F. Van Dijk, L. P. Barry, and A. J. Seeds, " $100 \mathrm{~Gb} / \mathrm{s}$ multicarrier THz wireless transmission system with high frequency stability based on a gain-switched laser comb source," IEEE Photonics J., vol. 7, no. 3, 2015.

[8] H.-J. Song, K. Ajito, Y. Muramoto, A. Wakatsuki, T. Nagatsuma, and N. Kukutsu, "Uni-travelling-carrier photodiode module generating $300 \mathrm{GHz}$ power greater than $1 \mathrm{~mW}$," IEEE Microw. Wirel. Components Lett., vol. 22, no. 7, pp. 363-365, 2012.

[9] IEEE 802.15 TG3d Technical Requirements Document, document number 14/0309r20, March 2016, downloaded from http://www.ieee802.org/15/pub/index_TG3d.html (accessed 19 September 2016).

[10] Y. Ou, S. Yan, A. Hammad, B. Guo, S. Peng, R. Nejabati, and D. Simeonidou, "Demonstration of virtualizeable and software-defined optical transceiver," J. Lightwave Technol., vol. 34, no. 8, pp. 19161924, 2016

[11] H. Shams, M. J. Fice, L. Gonzalez-Guerrero, C. C. Renaud, F. van Dijk, and A. J. Seeds, "Sub-THz wireless over fibre for frequency band $220 \mathrm{GHz}-280 \mathrm{GHz}$," to be published in J. Lightwave Technol., 2016.

[12] T. Shao, H. Shams, P. M. Anandarajah, M. J. Fice, C. C. Renaud, F. van Dijk, A. J. Seeds, and L. P. Barry, "Phase noise investigation of multicarrier sub- $\mathrm{THz}$ wireless transmission system based on an injection-locked gain-switched laser," IEEE Trans. Terahertz Sci. Technol., vol. 5, no. 4, pp. 590-597, Jul. 2015.

[13] H. Shams, M. J. Fice, K. Balakier, C. C. Renaud, F. van Dijk, and A. J. Seeds, "Photonic generation for multichannel $\mathrm{THz}$ wireless communication," Opt. Express, vol. 22, no. 19, pp. 23465-23472, 2014. 